\title{
Effect of graphene oxide (GO) and polyvinylpyrollidone (PVP) additives on the hydrophilicity of composite polyethersulfone (PES) membrane
}

\author{
Nurul Fattin Diana Junaidi a, Nur Hidayati Othman a, ${ }^{*}$, Munawar Zaman Shahruddin a, Nur \\ Hashimah Alias ${ }^{a}$, Woe Jye Lau ${ }^{\text {b }}$, Ahmad Fauzi Ismail ${ }^{\text {b }}$ \\ a Faculty of Chemical Engineering, Universiti Teknologi MARA 40450 Shah Alam Selangor Darul Ehsan, Malaysia \\ b Advanced Membrane Technology Research Centre (AMTEC), Universiti Teknologi Malaysia, 81310 Skudai, Johor, Malaysia \\ * Corresponding author: nurhidayati0955@salam.uitm.edu.my
}

\section{Article history}

Received 5 May 2018

Revised 1 June 2018

Accepted 2 July 2018

Published Online 25 June 2019

\section{Graphical abstract}

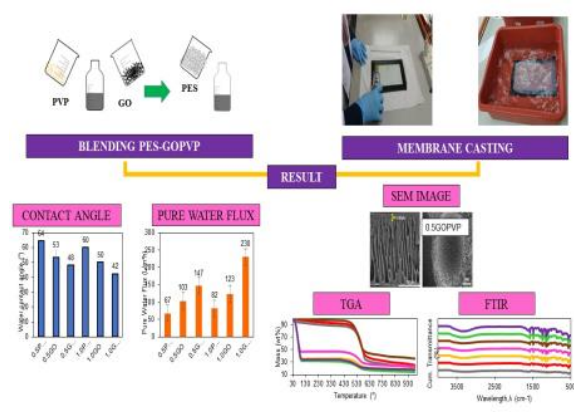

\begin{abstract}
Membrane based separation system is considered as a promising technology to purify water, owing to its simplicity and efficiency in operation. However, the application is limited by membrane fouling, which can lead to the declination of water flux and premature failure of membrane. The fouling can be controlled through membrane surface modification by blending hydrophilic materials during the casting solution preparation. Polyethersulfone (PES) membrane is naturally hydrophobic due to lack of oxygen functional group, which limits its application in the filtration of water. Therefore, modification of PES-based membranes is required. In this work, modification of the PES membrane was carried out by incorporating carbon-based nanomaterials (graphene oxide (GO)) and a well-known organic polymer (polyvinylpyrrolidone (PVP)). The effect of each additive toward the hydrophilicity of composite PES membrane was then investigated. GO was synthesized using modified Hummers method due to its simpler and shorter process. Each additive was added during the casting solution preparation and the amount added was varied from 0.5 to $1.0 \mathrm{wt} \%$. The resultant composite PES membranes were characterized using XRD, FTIR and TGA prior to hydrophilicity and pure water flux (PWF) measurement. It was observed that the additives (PVP and GO) have significantly affected the membranes hydrophilicity, resulting in lower contact angle and higher pure water flux. The highest value of PWF $\left(230 \mathrm{~L} / \mathrm{m}^{2} . h\right)$ with lowest contact angle $\left(42^{\circ}\right)$ were observed for PES-1.0GOPVP membrane due to high amount of GO and PVP. Improved PWF performance of composite PES-1.0GOPVP membrane was attributed to the better dispersibility of the PVP and GO and increased surface hydrophilicity of the modified composite membranes. This study indicated that PVP and $\mathrm{GO}$ are effective modifiers to enhance the performance of PES membrane.
\end{abstract}

Keywords: Graphene oxide, polyvinylpyrollidone, hydrophilicity, pure water flux, composite PES membrane

(C) 2019 Penerbit UTM Press. All rights reserved

\section{INTRODUCTION}

Membranes are thin and porous sheets of material that are able to separate contaminants from water when a driving force is applied. It is increasingly utilized for drinking water and wastewater treatment. The commonly used membranes in separation processes are ultrafiltration (UF) and microfiltration (MF) membranes that are made of ceramic and polymeric materials (Jamaly et al., 2015). The advantages of membrane technology are that it can work without addition of chemicals, easy to operate, high efficiency, energy and space saving and easy to scale up. Despite the many attractive advantages of membrane, some of the main drawbacks such as low permeation or rejection and fouling difficulties may limit its widespread application. It is also less robust and incapable of self-cleaning. Thus, chemical treatments are required to prolong membrane life (Thuyavan et al., 2016).

Polyethersulfone (PES) is used extensively in the membrane preparation due to its unique and excellent properties such as chemical resistance, good thermal stability, and high mechanical strength, enabling it to be broadly used for preparation of microfiltration (MF), ultrafiltration (UF) and gas separation membranes (Ghasem et al., 2012). However, Jin et al. (2013) stated that low flux and weak antifouling capability resulted from PES's poor hydrophilic property have limited the wide applications of PES membrane in wastewater treatment. This is proved by Rastegarpanah \& Mortaheb (2016), where they showed that PES membrane exhibits partial hydrophilic characteristics and cannot be functional directly in the membrane distillation due to the occurrence of etheric bonds in the PES chains. 
Kiran et al. (2015) also concluded that PES membranes have limitations as the inherent hydrophobic property that leads to fouling due to lack of oxygen functional group in PES structure. Thus, PES membrane needs to be modified. Blending the membrane with more hydrophilic nanomaterials has been revealed to combat this matter and since then, a number of hydrophilic nanomaterials has been investigated (Subramaniam et al., 2017). The blending of these additives is not only significantly improved the membrane hydrophilicity, but surface roughness, surface charge, and the pore size of membranes are also improved (Safarpour et al. 2015). As the final properties of membranes are relied on the properties of additives added into the membrane polymer matrix, it is important to understand the effects of additive types and concentrations, molecular weights of additives, and solvents used during the membrane fabrication towards the final performance of PES composite membrane (Prince, 2015). Chang et al. (2014) verified that the additives with the good water solubility can modify in/on membranes by hydrogen bonding. The addition of nanoparticles additives can also improve the final properties of the membranes such as the increase of permeability, selectivity, strength, and hydrophilicity (Yong et al., 2013).

Polyvinylpyrrolidone (PVP) is a hydrophilic polymer with no hydroxide group or ionic charged group. It has been widely used as a modifier for the alteration of PES membrane through the blending method (Zhao et al., 2011). The advantages of PVP are high chemical stability, nontoxicity and excellent solubility in many polar solvents (Chen et al., 2015). PVP also possesses good water solubility and hydrophilicity that suitable as pore-forming reagent to control the microstructure of membranes. PVP also can functions as antibiofouling modifier in order to enhance the surface hydrophilicity and thus, reducing fouling. Wang et al. (2009) agreed that PVP gives effect on membrane flux and rejection. Their results indicated that with the up to $23 \%$ as compared to pristine PES. Chang et al., (2014) proved that the incorporation of PVP with presence of GO can optimize membrane performance due to the formation of hydrogen bonds between GO and PVP.

Graphene oxide (GO) is a two-dimensional carbon material that received tremendous attentions owing to its fantastic chemical properties (Yu et al., 2013). GO exhibits similar properties as graphene but differs by having many oxygen-containing functional groups, for instance, epoxy, hydroxyl, carbonyl and carboxyl groups, on the edges and basal planes of the sheet. The hydrophilic groups enable stable dispersions of GO sheets in aqueous media, which provide a facile processing and stacking of these sheets. In addition, these functional groups allow reactive modifications of GO, by which one can readily control the microstructures and chemical properties of GO membranes, as well as develop GO-based hybrid membranes (Liu et al., 2015). GO can be produced via either Brodie, Staudenmaier or Hummers method, or via various modifications of these techniques (for example, the Hummer's modified method). However, modified Hummers method is usually preferred due to its simpler and shorter process (Hegab \& Zou, 2015). According to Chen et al. (2015), GO can be well dispersed in the PVP matrix, thus boosting its performance and characteristic.

The amount of nanomaterials added in the membranes is known to significantly affect the final membrane properties. High concentration of nanomaterials can lead to a reversible decrease of membrane pore size. So, the amount of additives needs to be controlled at optimum level to prevent this scenario from happening (Kaminska et al., 2015). In this work, polyvinylpyrrolidone (PVP) and graphene oxide (GO) were used as additives and the concentrations inside the casting solution were varied to understand their effects towards the properties and hydrophilicity of composite PES membrane.

\section{EXPERIMENTAL}

\section{Materials}

Graphite powders (MW=12.01 g/mol, COMAK) were used as raw material to synthesis GO. $\mathrm{H}_{2} \mathrm{SO}_{4}\left(98 \%\right.$, R\&M Chemicals), $\mathrm{NaNO}_{3}$ (84.99 g/mol, SYSTERM), $\mathrm{KMnO}_{4}(158.05 \mathrm{~g} / \mathrm{mol}, \mathrm{R} \& \mathrm{M}$ Chemicals), $\mathrm{H}_{2} \mathrm{O}_{2}$ (30\%, Merck), $\mathrm{HCl}$ (brand)) were used as received during the oxidation and exfoliation of GO. PES (MW=22,000 g/mol) and N, N Dimethylacetamide (DMAc) were purchased from Merck USA and used as a polymer and solvent in the preparation of solution cast membrane. Polyvinylpyrrolidone (PVP) from Fisher Scientific UK was used to enhance water hydrophilicity. Deionized water (DI) water was used during sample preparation and pure water flux (PWF) measurement.

\section{Synthesis of graphene oxide (GO)}

GO was prepared by using modified Hummer's method (Aditya Kiran et al., 2016). First, $5 \mathrm{~g}$ of graphite powder and $2.5 \mathrm{~g}$ of sodium hydroxide were added into $200 \mathrm{~mL}$ of concentrated sulphuric acid and stirred for $1 \mathrm{~h}$. Then, $30 \mathrm{~g}$ of potassium permanganate was added into the beaker and the solution was stirred for another $4 \mathrm{~h}$ at temperature below $15^{\circ} \mathrm{C}$. The mixture was then continuously stirred for $20 \mathrm{~h}$ at room temperature. Next, the mixture was slowly heated to $70^{\circ} \mathrm{C}$ for $2 \mathrm{~h}$ and $90{ }^{\circ} \mathrm{C}$ for another 1 hour with addition of $100 \mathrm{~mL}$ of distilled water. $\mathrm{H}_{2} \mathrm{O}_{2}$ was then used to stop the reaction. The solution was then washed using $\mathrm{HCl}$ solution for several times, followed by centrifugation. The $\mathrm{GO}$ was then dried for $24 \mathrm{~h}$ at $60-70{ }^{\circ} \mathrm{C}$ to form thin film GO. Finally, the dried GO film was purified using acetone, followed by vacuum filtration and drying at $60^{\circ} \mathrm{C}$ for $12 \mathrm{~h}$.

\section{Composite membrane fabrication}

The PES-GO and PES-GOPVP membranes were fabricated via phase inversion (immersion precipitation) method. The casting solution was prepared by using PES and DMAc as polymer and solvent, respectively. GO and PVP were used as hydrophilic modifiers/additives to improve performance of membrane. The addition of additives onto the PES membranes was carried out using blending technique. PES membrane was prepared and used as a control experiment. The membrane fabrication was further illustrated in Fig. 1.

Table 1 shows the formulation of composite PES membranes prepared in this work using GO and PVP additives.

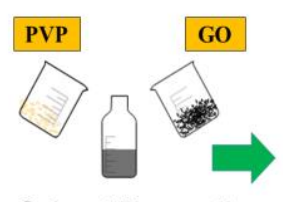

Solvent Preparation

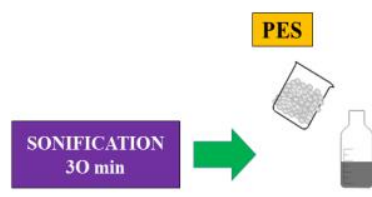

Polymer Addition
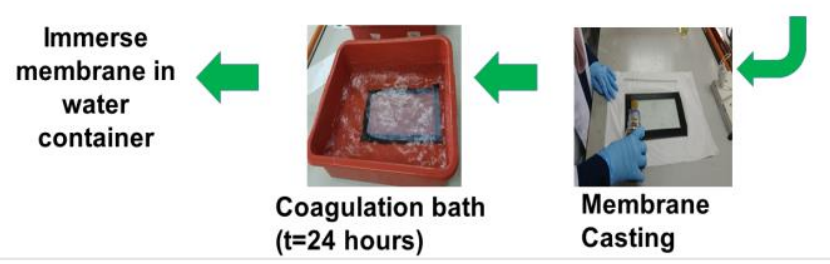

Fig. 1 Schematic diagram for preparation of composite PES-GO and PES-GOPVP membranes.

Table 1 Composite membrane composition.

\begin{tabular}{lcccc}
\multicolumn{1}{c}{ Membrane } & $\begin{array}{c}\text { PES } \\
\text { (wt \%) }\end{array}$ & $\begin{array}{c}\text { DMAc } \\
\text { (wt \%) }\end{array}$ & $\begin{array}{c}\text { GO } \\
\text { (wt \%) }\end{array}$ & $\begin{array}{c}\text { PVP } \\
\text { (wt \%) }\end{array}$ \\
\hline PES & 17 & 83 & - & - \\
PES-0.5GO & 17 & 82.5 & 0.5 & - \\
PES-0.5PVP & 17 & 82.5 & - & $0.5-$ \\
PES-0.5GOPVP & 17 & 82 & 0.5 & 0.5 \\
PES-1.0GO & 17 & 82.0 & 1.0 & - \\
PES-1.0PVP & 17 & 82.0 & - & 1.0 \\
PES-1.0GOPVP & 17 & 81 & 1.0 & 1.0 \\
\hline
\end{tabular}

\section{Characterization of GO and composite PES membrane}

X-Ray diffraction, XRD (Rigaku) analysis was first carried out to confirm the formation of graphene oxide from graphite. The measurement was performed in scanning range of $40 \mathrm{Kv}, 40 \mathrm{~mA}, 5-80$ ${ }^{\circ}$ with $2 \%$ min of scanning rate. The functional groups of GO were 
observed using Fourier Transform Infrared (FTIR, Perkin Elmer). The spectra were recorded in the wavelength range of $500-4000 \mathrm{~cm}^{-1}$. The samples were then characterized using thermogravimetric analysis (TGA) under $\mathrm{N}_{2}$ atmosphere at a temperature range between $50{ }^{\circ} \mathrm{C}$ to $1000{ }^{\circ} \mathrm{C}$, with a heating rate of $10{ }^{\circ} \mathrm{C} / \mathrm{min}$. The morphology of the membrane was then observed by using Scanning Electron Microscope, SEM (Hitachi, S-3400N). The hydrophilic behavior of membranes was tested using water contact angle goniometer (AST Product INC, VCA3000s).

Pure water flux (PWF) test was then conducted to determine the permeation of water. The test was carried for at room temperature and 3 bar. The flux was then calculated by using equation (1).

$$
J=\frac{V}{A \Delta t}
$$

Where $J$ is pure water flux $\left(\mathrm{L} / \mathrm{m}^{2} \mathrm{~h}\right), V$ is volume of permeate $(\mathrm{L})$, A is effective area of membrane $\left(\mathrm{m}^{2}\right)$ and $\Delta t$ is the sampling time $(\mathrm{h})$.

\section{RESULTS AND DISCUSSION}

\section{Characterization of GO}

XRD analysis was carried out to confirm the formation of GO from graphite. From Fig. 2 and Fig. 3, it can be observed that XRD spectra of graphite and GO showed a characteristic peak at around $2 \theta$ $=10.18$ respectively. The XRD pattern of graphite has a very intense and sharp peak, indicating that the graphite is highly crystalline and ordered structure. After the oxidation and exfoliation processes, the peaks disappeared and new peak arouse at lower degree $\left(10.18^{\circ}\right)$, confirming the formation of GO. The interlayer spacing obtained for graphite and GO were $0.324 \mathrm{~nm}$ and $0.868 \mathrm{~nm}$, respectively. It can be observed that after the oxidation process, GO possesses bigger interlayer space. According to Tissera et al. (2015), the high interlayer space in the synthesized GO is existed due to occurrence of oxygenated functional groups and interpolated water molecules in the graphite.

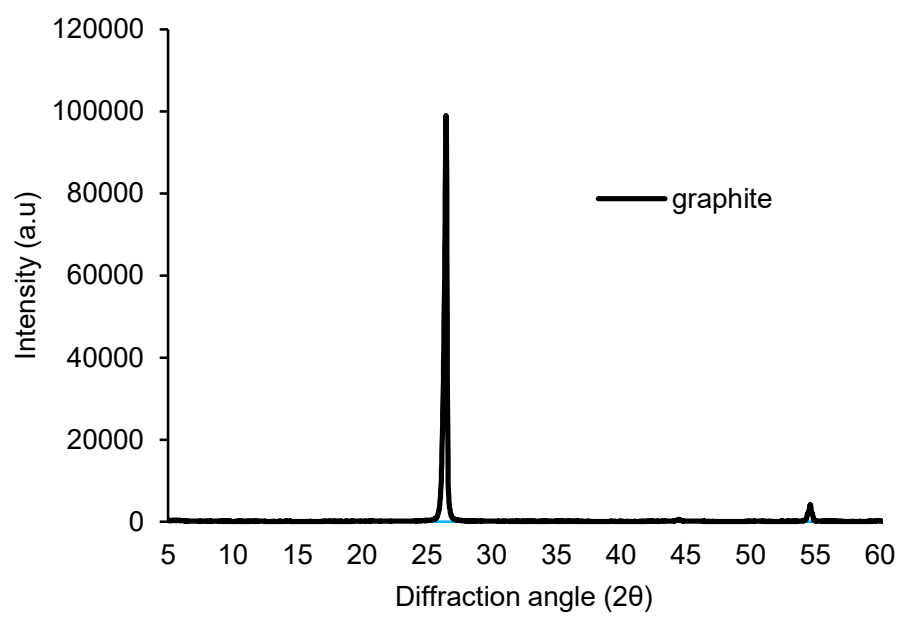

Fig. 2 XRD pattern for graphite.

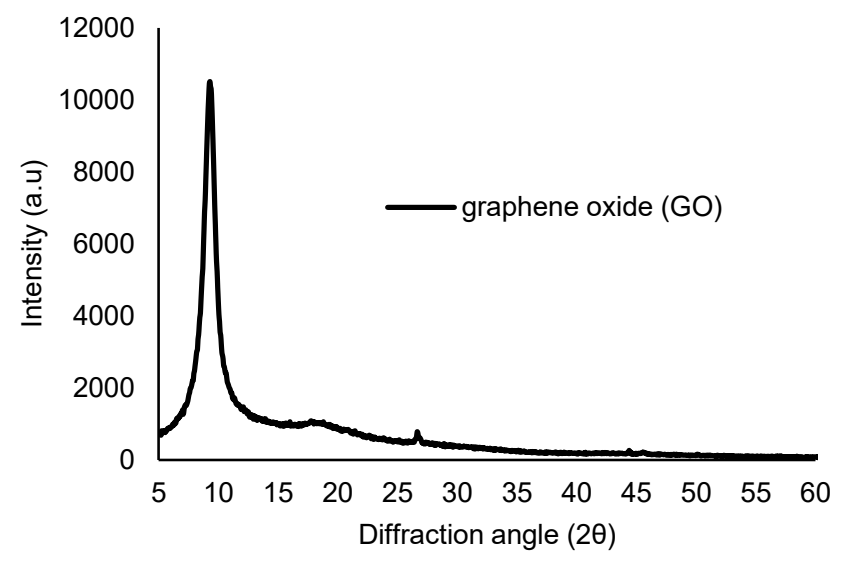

Fig. 3 XRD pattern for GO.

\section{Characterization of composite PES membrane}

Fourier transform infrared (FT-IR) test was carried out to characterize the composite PES membrane before and after addition of additives. The absorption spectral peaks at the wavelength of 3414 , $1556,1120 \mathrm{~cm}^{-1}$ in $\mathrm{GO}$ particles correspond to the functional groups of hydroxyl $(\mathrm{O}-\mathrm{H})$ and carboxyl group $(\mathrm{C}=\mathrm{O}$ and $\mathrm{C}-\mathrm{O})$, respectively. These functional groups can alter filtration application by improving its hydrophilic property of the membrane surface (Zinadini et al., 2014). The spectrum also showed two bands at $1078 \mathrm{~cm}^{-1}$ and $1234 \mathrm{~cm}^{-1}$ originated from the $\mathrm{C}=\mathrm{O}$ stretching vibrations of alkoxyl. After the introduction of GO, the spectra indicated the GO entrapped and formed as one part of PES structure which responsible for enhancing the hydrophilic characteristics of the membrane. This means that the carboxylic acid groups are formed on the surface of the graphene. The band at $1627 \mathrm{~cm}^{-1}$ is assigned to the vibrations of the adsorbed water molecules and the contributions from the vibration of aromatic $\mathrm{C}=\mathrm{C}$.

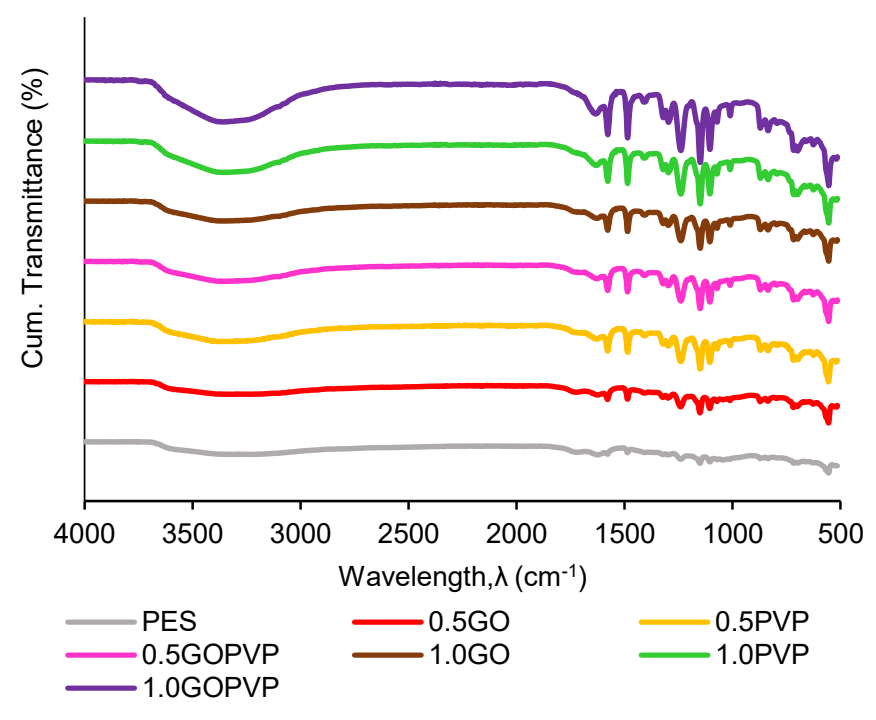

Fig. 4 FT-IR spectra of composite PES membrane. 
The TGA curves and decomposition temperature value of neat and composite PES membranes in the temperature range of $50-1000{ }^{\circ} \mathrm{C}$ were shown in Fig. 5. It can be seen that the composite PES membranes which contained PVP show a weight loss around $50 \%$ at $110^{\circ} \mathrm{C}$. These losses are corresponded to the removal of water and $\mathrm{COOH}$ groups, respectively. Plus, the PVP-composite membrane is sent for testing in wet condition. The high decrement of weight lost around $110^{\circ} \mathrm{C}$ is also a result of oxygen functional groups removal due to the decomposition of the hydroxyl or epoxy groups. PES membrane holds the decomposition temperature at around $405{ }^{\circ} \mathrm{C}$, corresponding to the degradation of the polymer backbone in both blend and bare membrane (PES). It is interesting to note that the decomposition temperature is improved when GO is incorporated into PES membranes compared to PVP Oh et al. (2014). From these results, it can be clearly seen that GO is effectively oxidized.

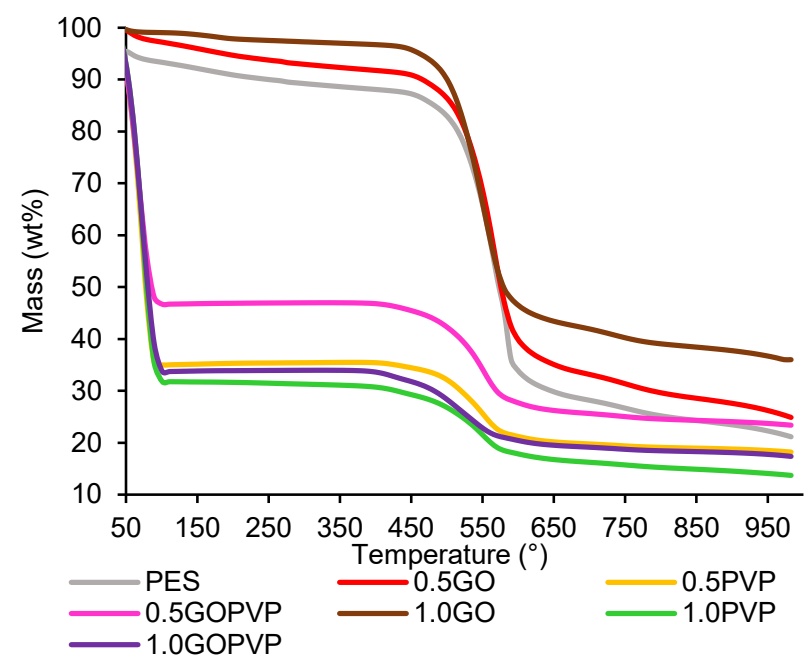

Fig. 5 Thermogravimetric analysis of composite PES membranes.

\section{Effect of GO and PVP additives on hydrophilicity and pure water flux (PWF)}

The hydrophilicity of the membrane surface can be measured by using water contact angle measurement (Ganesh et al., 2013). The water contact angle was measured by using the sessile drop method. A decreasing trend of contact angle was observed as the additives loading was increased in the composite PES membrane as shown in

Fig. 6. This lower contact angle means that the membrane surface is more attracted to water and hydrophilic in nature. In general, when the same amount of additives (PES-0.5PVP and PES-0.5GO) is used, the composite PES membrane that contained GO $\left(53^{\circ}\right)$ has lower contact angle that the one contained PVP $\left(64^{\circ}\right)$. This indicates that the surfaces of composite PES-GO membranes are relatively more hydrophilic compared to pristine PES membrane. During the membrane formation, the hydrophilic GO migrates towards the top surface of the membrane as the top layer is more exposed to water (nonsolvent), thus giving the appearance of dark color of the top surface in comparison to the bottom surface (Ganesh et al., 2013). However, the hydrophilicity of composite PES membrane can be further improved when both PVP and GO are used. By combining both additives (PES$0.5 \mathrm{GOPVP})$, more hydrophilic membrane can be achieved $\left(48^{\circ}\right)$. Other than additive types, the amount of additive also plays a significant role. By increasing the amount of additive from $0.5 \mathrm{wt} \%$ to $1.0 \mathrm{wt} \%$, the hydrophilicity of composite PES membranes increased significantly. The lowest contact angle $\left(42^{\circ}\right)$ was observed for PES membrane due to the absence of additives to alter membrane pore size. Membrane with no additive will exhibit more dense and closed pack top layer pore as illustrated in SEM image of Fig 8. The membrane pore size and distribution, and the membrane hydrophilicity had great effect on the membrane flux. With the increase of the membrane pore size and hydrophilicity, the flux is increased with lower contact angle (Zhao et al., 2011).

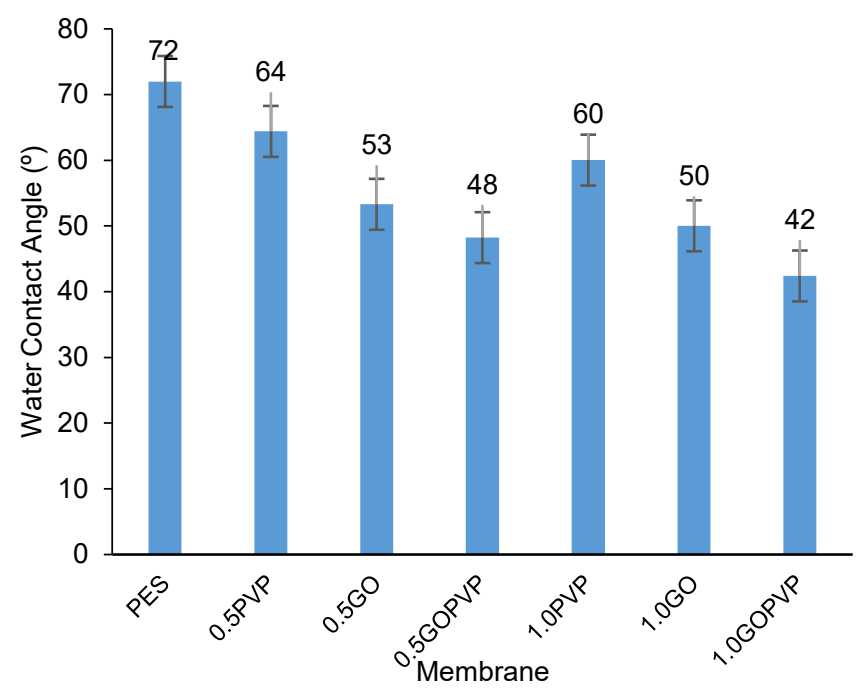

Fig. 6 Water contact angle of composite PES membrane.

Fig. 7 shows the pure water flux (PWF) for PES and composite PES membranes. It can be seen that the trend of PWF for composite PES membrane is inversely proportional with water contact angle. Composite PES membranes that contained GO have higher PWF compared to PVP. When GO and PVP are both added, the PWF increases significantly compared to when associated with bare PES membrane. The PWF of composite PES membrane with addition of modifier GO or PVP experienced an increment about more than $40 \%$ compared to bare PES membrane. The value of PWF for PES$0.5 \mathrm{GOPVP}$ was $147 \mathrm{~L} / \mathrm{m}^{2} \mathrm{~h}$ and by increasing the value of additives to $1.0 \mathrm{wt} \%$, the value of PWF became almost double to $230 \mathrm{~L} / \mathrm{m}^{2} \mathrm{~h}$. PVP is not only known to help increasing the hydrophilicity of polymeric membrane, but it also can alter the microstructure of the membranes. A number of studies showed that PVP can be used as a pore filler to enhance the micro voids of membrane. This study established the importance of using both additives to enhance the hydrophilicity of composite PES membrane. The synergetic effects between GO and PVP improve the hydrophilicity due to hydroxyl functional groups in GO and water soluble PVP that acts as pore-forming reagents (Chang et al., 2014). The main factor is that GO particles with oxygencontaining groups dispersed in the casting solution can homogeneously distribute on the membrane surface or membrane pores during the phase inversion process with the aid of hydrogen bonds between GO and PVP, as shown in Fig 8.

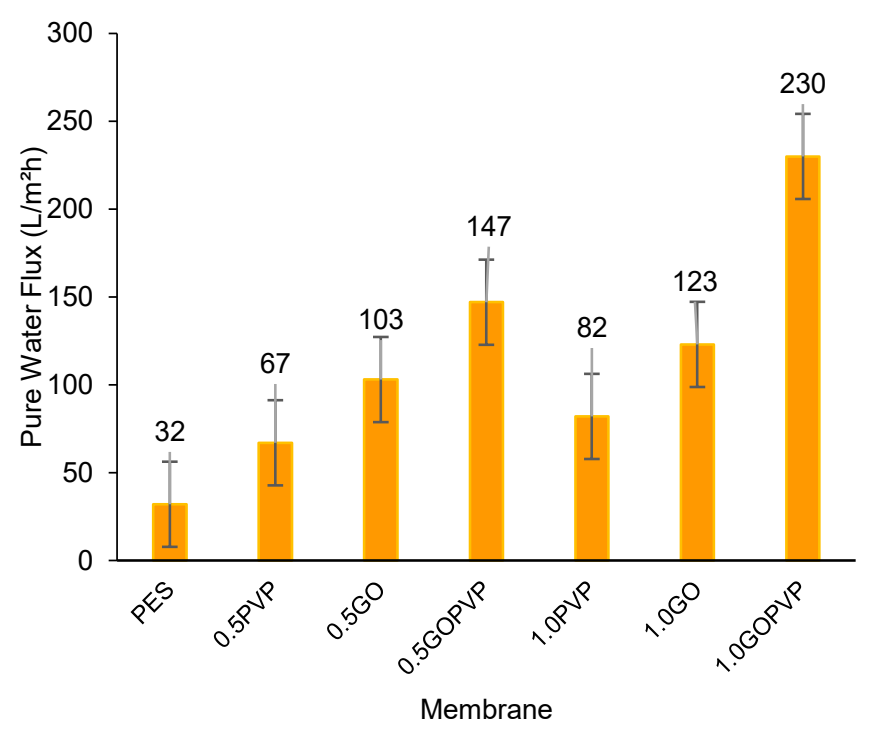

Fig. 7 Pure water flux of composite PES membranes. 
Fig 8 shows the SEM images of PES, PES-0.5GO, PES-0.5PVP and PES-0.5GOPVP composite PES membranes. The cross-sectional SEM images of the prepared membranes displayed typical characteristic of asymmetric porous structure with a dense skin toplayer followed by a finger-like porous sub-layer. It was found that the top surface contained much smaller pore size compared to the bottom micro void structure. This can be due to the formation of skin layer that induced by phase inversion process (Sirinupong et al., 2017). From Fig 8 , the SEM image of bare PES membrane showed denser top layer pore opening compared to PES membrane with fusion of additives. Consequently, due to the carbon-based structure of GO and PES polymer, the GO was well spread in the polymer and agglomeration on the surface was not observed. The finger-like pores for the PVP embedded membranes were observed to be slightly wider than the one with GO. This is because of the hydrophilic nature of GO increases the mass transfer rate between the solvent and the non-solvent during phase inversion (Rezaee et al., 2015). From the PWF and contact angle studies, it is observed that the addition of GO can significantly affect the hydrophilicity of the PES membrane due to presence of hydroxyl and carbonyl functional group in GO. Besides, the presence of PVP in the cast solution also facilitates water diffusion to the polymer cast film, causing faster solvent and non-solvent (water) exchange rate during phase inversion process and leading to formation of long finger-like voids.(Sirinupong et al., 2017)
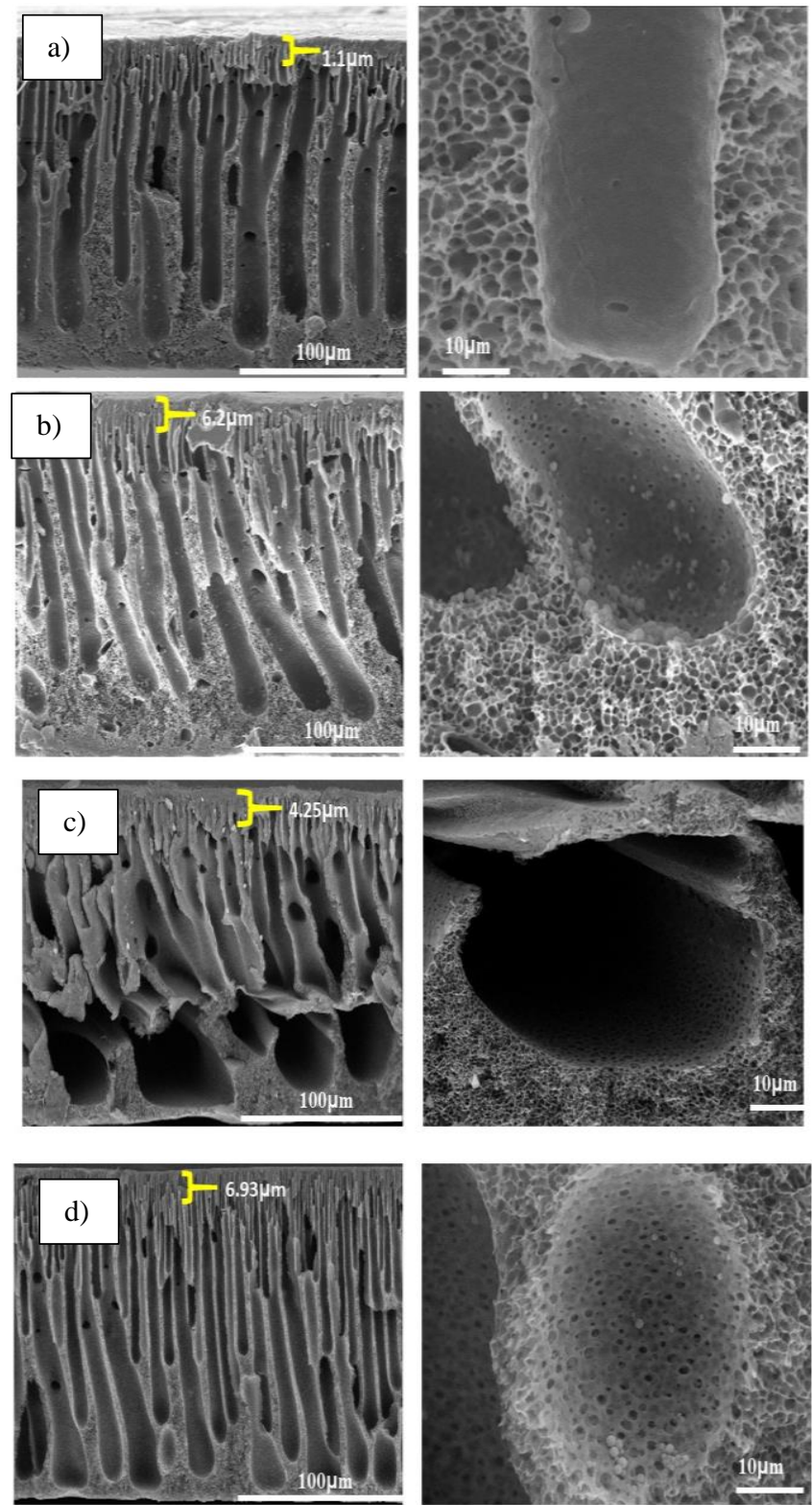

Fig 8. SEM images of a) PES, b) 0.5GO, c) 0.5PVP, d) 0.5GOPVP.

\section{CONCLUSION}

Composite PES membranes were prepared by using blending method. Different types (GO and PVP) and amounts of additives $(0.5$ and $1.0 \mathrm{wt} \%$ ) were used in this study and their effects on the hydrophilicity and morphology of composite PES membranes were examined by water contact angle and PWF. The prepared composite PES membranes were first analyzed using FTIR and TGA. The results showed reduction of water contact angle and higher PWF performance accomplished. The SEM images showed that the prepared composite membranes were in asymmetric structure with dense top layer and supported by finger-like structure. When higher amount of GO and PVP was used, the finger-like structure became bigger. This might be the reason behind higher PWF value. The highest value of PWF (230 $\mathrm{L} / \mathrm{m}^{2} . \mathrm{h}$ ) with lowest contact angle $\left(42^{\circ}\right)$ were observed for PES1.0GOPVP membrane due to high amount of GO and PVP. GO and PVP as pore filler altered membrane structure to become more microporous, less dense on top layer and more straight fingerlike structure that allow water channeling to be faster. This study established the importance of using both additives to enhance the hydrophilicity of composite PES membrane and improve the antifouling properties of the membranes.

\section{ACKNOWLEDGEMENT}

The authors would like to acknowledge Ministry of Higher Education (MOHE) and Universiti Teknologi MARA for the FRGS research funding (FRGS/ 1/ 2015/ TK05/ UITM/ 03/2), which made this important research viable and effective.

\section{REFERENCES}

Aditya Kiran, S., Lukka Thuyavan, Y., Arthanareeswaran, G., Matsuura, T., Ismail, A. F. 2016. Impact of graphene oxide embedded polyethersulfone membranes for the effective treatment of distillery effluent. Chemical Engineering Journal, 286, pp. 528-537.

Chang, X., Wang, Z., Quan, S., Xu, Y., Jiang, Z., Shao, L. 2014. Exploring the synergetic effects of graphene oxide (GO) and polyvinylpyrrodione (PVP) on poly (vinylylidenefluoride) (PVDF) ultrafiltration Membrane performance. Applied Surface Science, 316(1), pp. 537-548.

Chen, S., Cheng, B., Ding, C. 2015. Synthesis and characterization of poly(vinyl pyrrolidone)/reduced graphexide nanocne oomposite. Journal of Macromolecular Science, Part B: Physics, 54(4), pp. 481-491.

Ganesh, B. M., Isloor, A. M., Ismail, A. F. 2013. Enhanced hydrophilicity and salt rejection study of graphene oxide-polysulfone mixed matrix membrane. Desalination, 313, pp. 199-207.

Ghasem, N., Al-Marzouqi, M., Zhu, L. 2012. Preparation and properties of polyethersulfone hollow fiber membranes with o-xylene as an additive used in membrane contactors for $\mathrm{CO}_{2}$ absorption. Separation and Purification Technology, 92, pp. 1-10.

Hegab, H. M., Zou, L. 2015. Graphene oxide-assisted membranes : Fabrication and potential applications in desalination and water puri fi cation. Journal of Membrane Science, 484, pp. 95-106.

Jamaly, S., Giwa, A., Hasan, S. W. 2015. Recent improvements in oily wastewater treatment: Progress, challenges, and future opportunities. Journal of Environmental Sciences (China), 37, pp. 15-30.

Jin, F., Lv, W., Zhang, C., Li, Z., Su, R., Qi, W., Yang, Q., He, Z. 2013. Highperformance ultrafiltration membranes based on polyethersulfonegraphene oxide composites. RSC Advances, 3, pp. 21394-21397.

Kaminska, G., Bohdziewicz, J., Calvo, J. I., Prádanos, P., Palacio, L., Hernández, A. 2015. Fabrication and characterization of polyethersulfone nanocomposite membranes for the removal of endocrine disrupting micropollutants from wastewater. Mechanisms and performance. Journal of Membrane Science, 493, pp. 66-79.

Kiran, S. A., Thuyavan, Y. L., Arthanareeswaran, G., Matsuura, T., Ismail, A. F. 2015. Impact of graphene oxide embedded polyethersulfone membranes for the ef- fective treatment of distillery effluen. Chemical Engineering Journal, pp. 1-42.

Li, F., Wang, W., Ren, Q. 2009. Preparation of Hollow fibre composite reverse osmosis membrane. Modern Applied Science, 4(1), pp. 82-90.

Liu, Y., Zhou, J., Zhu, E., Tang, J., Liu, X., Tang, W. 2015. Covalently intercalated graphene oxide for oil-water separation. Carbon, 82(C), pp. 264-272. 
Oh, Y. J., Yoo, J. J., Kim, Y. Il, Yoon, J. K., Yoon, H. N., Kim, J. H., Park, S. Bin. 2014. Oxygen functional groups and electrochemical capacitive behavior of incompletely reduced graphene oxides as a thin-film electrode of supercapacitor. Electrochimica Acta, pp. 118-128.

Prince, J. A. 2016. Design and development of biologically inspired materialsbased ultrafiltration membranes with enhanced antifouling property (Doctoral dissertation, Newcastle University)

Rastegarpanah, A., Mortaheb, H. R. 2016. Surface treatment of polyethersulfone membranes for applying in desalination by direct contact membrane distillation. Desalination, 377, pp. 99-107.

Rezaee, R., Nasseri, S., Mahvi, A. H., Nabizadeh, R., Mousavi, S. A., Rashidi, A., Jafari, A., Nazmara, S. 2015. Fabrication and characterization of a polysulfone-graphene oxide nanocomposite membrane for arsenate rejection from water. Journal of Environmental Health Science and Engineering, 13(16), pp. 1-11.

Safarpour, M., Vatanpour, V., Khataee, A. 2015. Preparation and characterization of graphene oxide/ $\mathrm{TiO}_{2}$ blended PES nano filtration membrane with improved antifouling and separation performance. Desalination, 393, pp.65-78.

Sirinupong, T., Youravong, W., Tirawat, D., Lau, W. J., Lai, G. S., Ismail, A. F. 2017. Synthesis and characterization of thin film composite membranes made of $\mathrm{PSF}_{-} \mathrm{TiO}_{2} / \mathrm{GO}$ nanocomposite substrate for forward osmosis applications. Arabian Journal of Chemistry, 11(7), pp.1144-1153.

Subramaniam, M. N., Goh, P. S., Lau, W. J., Tan, Y. H., Ng, B. C., Ismail, A. F. 2017. Hydrophilic hollow fiber PVDF ultrafiltration membrane incorporated with titanate nanotubes for decolourization of aerobicallytreated palm oil mill effluent. Chemical Engineering Journal, 316, pp. 101110.

Thuyavan, Y. L., Anantharaman, N., Arthanareeswaran, G., Ismail, A. F. 2016 Impact of solvents and process conditions on the formation of polyethersulfone membranes and its fouling behavior in lake water filtration. Journal of Chemical Technology and Biotechnology, 91(10), pp. $2568-2581$.

Tissera, N. D., Wijesena, R. N., Perera, J. R., Silva, K. M. N. De, Amaratunge, G. A. J. 2015. Hydrophobic cotton textile surfaces using an amphiphilic graphene oxide (GO) coating. Applied Surface Science, 324, pp. 455-463.

Yu, L., Zhang, Y., Zhang, B., Liu, J., Zhang, H., Song, C., Zhang, Y., Zhang, B., Liu, J., Song, C. 2013. Preparation and characterization of HPEI-GO/ PES ultrafiltration membrane with antifouling and antibacterial properties. Journal of Membrane Science, 447, pp. 452-462.

Zhao, W., Huang, J., Fang, B., Nie, S., Yi, N., Su, B., Li, H., Zhao, C. 2011. Modification of polyethersulfone membrane by blending semiinterpenetrating network polymeric nanoparticles. Journal of Membrane Science, 369(1-2), pp. 258-266.

Zinadini, S., Zinatizadeh, A. A., Rahimi, M., Vatanpour, V., Zangeneh, H. 2014. Preparation of a novel antifouling mixed matrix PES membrane by embedding graphene oxide nanoplates. Journal of Membrane Science, 453 , pp. 292-301. 